\title{
Personnel Scheduling: Comparative Study of Backtracking Approaches and Genetic Algorithms
}

\author{
Amol C. Adamuthe \\ Assistant Professor, Dept. of CSE, \\ Rajarambapu Institute of Technology \\ Islampur, MS, India.
}

\author{
Rajankumar S. Bichkar \\ Professor, Dept. of E\&TC Engg, \\ G. H. Raisoni College of Engg. \& Mgmt, \\ Pune, MS, India.
}

\begin{abstract}
Scheduling and timetabling problems are multi-constrained constraint satisfaction problems that have huge search space. These problems are NP hard. This paper investigates the use of backtracking approaches to laboratory personnel scheduling problem in which the objective is to assign tasks to employees. The main objective of this work is to search for better solutions than those obtained by authors using genetic algorithmic approach. The performance of backtracking algorithms is tested for different variable orderings, value ordering and consistency enforcement techniques. It is observed that the variable and value ordering backtracking with consistency enforcement techniques gives better results than the chronological backtracking as well as the results reported in the literature. This work indicates that the problem instance under consideration might have even better solutions which can possibly be obtained by suitably modifying the genetic algorithmic approach used earlier by authors or by using other optimization techniques such as simulated annealing or Tabu search.
\end{abstract}

\section{General Terms}

Algorithms, Combinatorial optimization, Scheduling problems, Backtracking.

\section{Keywords}

Personnel scheduling, Timetabling, Backtracking, Value ordering, Variable ordering, Consistency enforcement techniques. Genetic algorithms.

\section{INTRODUCTION}

Personnel scheduling problems are often encountered in service organizations such as call centers, airport ground, security agencies, hospitals, railway and bus. Causmaecker et al. [1] have classified personnel scheduling problem in four planning categories namely, permanence centered, mobility centered, fluctuation centered and project centered planning. The main difficulties in solving these problems are their highly constrained nature and the environmental conditions that are different for each organization such as working hours, planning periods, existence of breaks for employees, existence of parttime employees in addition to full-time ones etc.

Ernst et al. [2] presented review of 700 papers published since 1950 on personnel scheduling and rostering problems. They categorized papers according to the type of problem addressed, the application areas covered and the methods used for solution [2]. The application areas such as buses, nurse scheduling and airlines have got more attention from researchers in last few decades. Ernst et al. [3] have also presented review on staff scheduling and rostering with respect to applications, methods and models. Burke et al. [4] has given detailed survey on nurse rostering problem with respect to terminologies used, solution approaches and key issues in nurse rostering.

In literature, the terms scheduling and timetabling are used interchangeably. According to Wren [5]:
Scheduling is the allocation, subject to constraints, of resources to objects being placed in space-time, in such a way as to minimize the total cost of some set of the resources used.
Timetabling is the allocation, subject to constraints, of given resources to objects being placed in space- time, in such a way as to satisfy as nearly as possible a set of desirable objectives.

The common examples of scheduling are driver scheduling $[6,7]$ which seek to minimize the total cost and job shop scheduling $[8,9,10]$ which may seek to minimize either the number of time periods or some physical resources used. The examples of timetabling are class timetabling $[11,12]$ and examination timetabling [13] and some forms of personnel allocation.

Scheduling problems are special types of constraint satisfaction problems (CSPs) [14]. The constraint satisfaction problems consist of a set of variables, a finite and discrete domain for each variable from the set and a set of constraints. Each constraint is defined over some subset from the original set of variables and limits the combinations of values that the variables in this subset can take. The solution to CSP is to find assignment to all the variables such that it satisfies all the constraints specified. In some problems, the goal is to find all such solutions or an optimal solution. When the goal is to obtain an optimal solution over a given objective function, the CSP is called as constraint optimization problem [14, 15].

A variety of approaches can be used to solve CSPs. The integer programming techniques provide an exact solution whereas techniques such as simulated annealing, hill climbing, Tabu search, genetic algorithms and neural networks provide approximate solutions. Backtracking is one of the most general algorithm design technique [16] and is an effective technique to solve different constraint satisfaction problems [17, 18, 19, $20,21]$. It is an exhaustive search and guarantees the feasible and optimal solutions. However, as it checks all possible combinations of values for the assignments to variables, the time required to get the answer is unpredictable. Tree search combined with backtracking and consistency checking is a 
special-purpose technique that is widely used for solving CSPs [22]. Vipin Kumar [14], Roman Bartak [15], Dechter [17] and Brailsford et al. [22] have provided survey of backtracking approaches for solving constraint satisfaction problems.

In this paper, we have applied various backtracking techniques to the problem of assignment of tasks to the personnel in a laboratory. We have tested different variable and value ordering approaches with consistency enforcement techniques on two solution representations. The main objective of this paper is to search for better solutions to a specific instance of this problem [23] than those obtained by us using genetic algorithmic approach in [26]. This will provide further directions for improving the effectiveness of our genetic algorithm implementation to solve more complex problem instances.

The rest of the paper is organized as follows: In Section 2, different backtracking algorithms are described. The personnel scheduling problem is then explained in Section 3. The implementation details that include solution representations, backtracking algorithms, variable and value orderings and consistency enforcement techniques are described in Section 4. The results and discussion are presented in Section 5. It provides detailed description of the problem instance, results obtained and comparison with best results available in literature. Finally, the conclusion of our study is outlined in Section 6.

\section{BACKTRACKING APPROACHES}

Backtracking, first named by D. H. Lehmer, is a well-known algorithm design technique for the solution of a class of problems that deal with searching for a set of feasible solutions or an optimal solution satisfying some constraints [16]. It incrementally attempts to extend a partial solution that specifies consistent values for some of the variables, towards a complete assignment by repeatedly choosing a value for another variable consistent with the values in the current partial solution [15]. A general algorithm for backtracking [16] is given below.

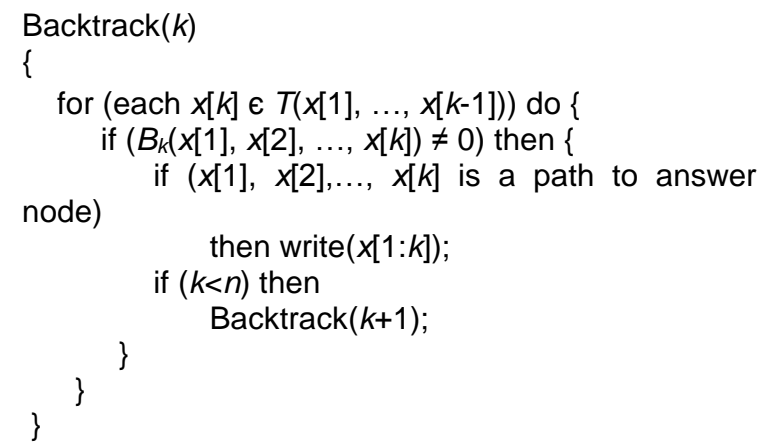

In this algorithm, $T$ is a function that accepts a partial solution to the problem $(x[1], \ldots, x[k-1])$ and returns a set of values consistent with this partial solution for variable $x[k]$. Also, $B_{k}$ is a bounding function that returns a non-zero value if the newly formed partial solution i. e. $x[1], x[2], \ldots, x[k]$, can lead to a feasible solution to the problem.

The chronological backtracking is a simple implementation of backtracking in which the algorithm always chronologically backtracks to the most recently instantiated variable. Thus, the search is similar to a tree traversal. The major problems with this approach are lot of redundant work and late detection of conflicts. This often leads to poor performance and thrashing.

Several variations of chronological backtracking algorithm are proposed in the past to speed up the search process. These include backjumping, backmarking and forward checking. They are explained below in brief. Kondrak [23] has provided more details about these techniques.

Instead of backtracking to the most recent variable, the backjumping approach can skip multiple variables and backjump to the highest variable that conflicted to the current variable. However, it buckjumps only from dead ends. Two common variations of backjumping are conflict directed backjumping and graph based backjumping. The conflict directed backjumping has an ability to perform multiple backjumps. After the initial backjump from a dead end it can continue backjumping across conflicts, which often results in significant saving of computation. The graph-directed backjumping also attempts to backtrack more than one level if possible. It utilizes knowledge about the constraint graph to backtrack to the highest variable connected to the current one.

The backmarking approach imposes a marking scheme on the chronological backtracking algorithm in order to eliminate some redundant consistency checks.

In contrast to above approaches which perform consistency checks backwards, forward checking performs consistency check forward that is between the current variable and the future variables.

Several researchers have further improved these approaches. Prosser [24, 25] has proposed hybrid backtracking algorithms by combining two or more basic algorithms. Kondrak [23] has proposed theoretical method for evaluation of selected basic and hybrid backtracking algorithms. Bartak [15] has reported different search techniques for solving constraint satisfaction problem such as systematic search algorithm, consistency techniques, constraint propagation, variable and value ordering and reducing search.

In order to increase the efficiency of chronological backtracking algorithm researchers have focused on following questions.

1. Which variable should be assigned next?

Several researchers have remarked that the order of instantiation of variables can have substantial impact on the search efficiency [15]. The variable ordering can be either static or dynamic. As the name indicates, in static variable ordering, the search order is fixed. It is specified before the search begins and is not changed thereafter. On the other hand, the dynamic variables ordering is more flexible. It allows the choice of next variable to be decided during the search process. However, dynamic ordering is not feasible for all tree search algorithms [22]. Some of the variable ordering heuristics reported in literature are given below.

- Select difficult variables prior to simple variables.

- Select variable with the fewest legal values, referred to as the minimum remaining values (MRV) heuristic.

- Select variable with the most constraints on remaining variables, referred to as degree heuristic.

2. In what order should values of variables be tried?

The order in which values for assignments are considered can also have substantial impact on the time to find the 
first feasible solution. The value ordering affects the solution tree arrangement. It has an advantage if it ensures that a branch, which leads to a solution, is searched earlier than branches leading to dead ends. For example, if the CSP has a solution, and if a correct value is chosen for each variable, then a solution can be found without backtracking [15]. Different variable ordering heuristics are reported in literature such as:

- Good value ordering heuristics reduce backtracking by selecting values that are expected to participate in a large number of solutions

- Least constraining value heuristic rules out the fewest values in the remaining variables.

3. Can we detect inevitable failure early?

This approach keeps track of remaining legal values for unassigned variables that are connected to current variable and the search is terminated when all such legal values are exhausted.

\section{PERSONNEL SCHEDULING PROBLEM}

Franses and Post [26] have described the laboratory personnel scheduling problem. It is an assignment problem in which the required numbers of tasks are known in advance and the employees are to be assigned to tasks by satisfying certain constraints. The solution of this problem requires consideration of the following characteristics of personnel and tasks.

1) Characteristics of Personnel:

- Skillset,

- Work regulations (full time or part time employee), and

- Availability on planning period,

2) Characteristics of Tasks:

- Category (half day, day task or week task),

- Priority,

- Requirement on planning period,

- Dependency with other tasks, and

- Rotation of tasks among personnel.

\subsection{Hard Constraints}

Hard constraints are those that must be satisfied. Violation of these constraints (also called as conflicts) will cause the solution to be infeasible. The following hard constraints are considered:

1) Coverage constraint: Every task must be allotted the required number of personnel.

2) Constraints by work regulations: Number of work hours assigned to personnel must satisfy his/her work regulations.

3) Skill set constraint: A task should not be assigned to an employee who is not skilled for it.

4) Constraints defined by task types: Tasks are categorized into three types namely, half-day task, day task and week task. If the task is week task then it must be assigned to same person for the entire week. Whereas, day task is given to same person in both slots (morning and evening) and half day task is to be assigned to different personnel during morning and afternoon slots of a day.

\subsection{Soft Constraints}

Soft constraints are those that are desirable in order to produce a good quality schedule but violations are allowed to satisfy hard constraints. In this problem, it is desired that the task assignment should be according to the expertise of employees. We have used task history cost i. e. the number of hours an employee has worked on a task, as his/her expertise for that task.

\section{BACKTRACKING APPROACHES FOR PERSONNEL SCHEDULING PROBLEM}

This section gives details about the solution representations, consistency enforcement backtracking algorithm, variable ordering and value ordering for personnel scheduling problem described in Section 3.

\subsection{Solution Representations}

The backtracking algorithms are applied to two different solution representations shown in Fig. 1 and Fig. 2. In Personnel-oriented solution representation shown in Fig. 1, a row represents a personnel and a column represents one day of planning period. The cells contain the task. For example, task $\mathrm{T}_{3}$ is assigned to personnel $\mathrm{P}_{2}$ on day 1 . In Task-oriented solution representation shown in Fig. 2, a row represents a task and a column represents one day of planning period. The cells contain the employees. For example, personnel $\mathrm{P}_{2}$ is assigned to task $T_{2}$ on day 1 .

\begin{tabular}{|c|c|c|c|}
\hline \multirow{2}{*}{ Personnel } & \multicolumn{3}{|c|}{ Tasks } \\
\cline { 2 - 4 } & Day 1 & $\ldots$ & Day D \\
\hline $\mathrm{P}_{1}$ & $\mathrm{~T}_{1}$ & $\ldots$ & $\mathrm{T}_{4}$ \\
\hline $\mathrm{P}_{2}$ & $\mathrm{~T}_{3}$ & $\ldots$ & $\mathrm{T}_{5}$ \\
\hline$:$ & & $\ldots$ & \\
\hline $\mathrm{P}_{\mathrm{i}}$ & $\mathrm{T}_{5}$ & $\ldots$ & $\mathrm{T}_{3}$ \\
\hline$:$ & & $\ldots$ & \\
\hline $\mathrm{P}_{\mathrm{N}-1}$ & $\mathrm{~T}_{2}$ & $\ldots$ & $\mathrm{T}_{2}$ \\
\hline $\mathrm{P}_{\mathrm{N}}$ & $\mathrm{T}_{8}$ & $\ldots$ & $\mathrm{T}_{1}$ \\
\hline
\end{tabular}

Fig. 1 The Personnel-oriented solution representation.

\begin{tabular}{|c|c|c|c|}
\hline \multirow{2}{*}{ Task } & \multicolumn{3}{|c|}{ Personnel } \\
\cline { 2 - 4 } & Day 1 & $\ldots$ & Day D \\
\hline $\mathrm{T}_{1}$ & $\mathrm{P}_{1}$ & $\ldots$ & $\mathrm{P}_{4}$ \\
\hline $\mathrm{T}_{2}$ & $\mathrm{P}_{2}$ & $\ldots$ & $\mathrm{P}_{5}$ \\
\hline$:$ & & $\ldots$ & \\
\hline $\mathrm{T}_{\mathrm{i}}$ & $\mathrm{P}_{5}$ & $\ldots$ & $\mathrm{P}_{3}$ \\
\hline$:$ & & $\ldots$ & \\
\hline $\mathrm{T}_{\mathrm{M}-1}$ & $\mathrm{P}_{2}$ & $\ldots$ & $\mathrm{P}_{2}$ \\
\hline $\mathrm{T}_{\mathrm{M}}$ & $\mathrm{P}_{8}$ & $\ldots$ & $\mathrm{P}_{1}$ \\
\hline
\end{tabular}

Fig. 2 The Task-oriented solution representation.

\subsection{Variable and Value Orderings}

We have implemented static variable ordering method for both the solution representations. 
- The first variable ordering is based on assignment completion for each day. Thus, the values are assigned to solution from top to bottom and from left to right.

- The second variable ordering, on the other hand, is based on assignment completion for an individual employee or a task. Thus, the assignments are done from left to right and from top to bottom.

Two value orderings are employed for assigning employees to tasks by considering the workload and/or skillset of employees as follows:

- The employees are considered in decreasing order of workload.

- The employees are considered in decreasing order of skillset and workload. The employee priorities are calculated based on employee work regulations (full timer and part timer) and number of tasks an employee can do.

While assigning tasks to employees, they are considered in decreasing order of skillset (history cost). This increases the possibility of better assignments with respect to history cost.

\subsection{Consistency Enforcement Techniques}

A consistency enforcement backtracking algorithm [27] is given below for personnel scheduling problem.

1. If all assignments have been scheduled then stop.

2. Apply consistency enforcing procedure

3. If a dead end is detected then backtrack (i. e. select an alternative if available and go back to step 1) else stop and report that the problem is infeasible

4. Select next assignment to be scheduled (variable ordering)

5. Select a promising value for that assignment (value ordering)

6. Create a new search state by adding new reservation assignment to the current partial schedule. Go back to 1 .

The consistency enforcement techniques prune the search space by eliminating local inconsistencies that cannot participate in a global solution [28]. These techniques transform the problem into an equivalent but more explicit form [17]. To solve personnel scheduling problem, consistency enforcement techniques are introduced by adding new constraints and removing values from variable domains.

\section{RESULTS AND DISCUSSION}

We have solved the problem instance given by Franses and Post [26] with small variation. A laboratory has seven different tasks that should be assigned to personnel every day during the planning period. The tasks are named as AF, CT, IN, KB, PB, $\mathrm{PR}$ and TF. All tasks are treated as week tasks. Thus, tasks must be allocated to same personnel during a week. Two persons are required to carry out tasks $\mathrm{PB}$ and $\mathrm{TF}$, whereas, all other tasks require only one person.

The laboratory has 14 employees and works in two shifts, morning and afternoon. Two types of work regulations are under consideration, fulltime employees who work 40 hours per week and part time employees who work 16 or 24 hours in a week. We have assumed that each employee is available on all working days and that the part time employees will work only on limited number of days.
The number of days which an employee can be assigned work during the planning period is shown in Table 1. Thus, employees A, E, F, L and $\mathrm{M}$ will work on 3 days per week whereas, employees $\mathrm{C}, \mathrm{D}, \mathrm{I}, \mathrm{J}$ and $\mathrm{N}$ will work on 2 days per week. Employees B, G, H and K are fulltime employees. Table 1 also shows history costs i. e. the number of hours worked by employees on each task during last 15 weeks. History cost represents an employee's expertise as well as willingness to do specific tasks. Thus, the history costs are considered as skillset for employees.

Table 1 History cost (skillset) data used by Franses and Post [26].

\begin{tabular}{ccccccccc}
\hline \hline & $\boldsymbol{n}^{\dagger}$ & $\mathbf{A F}$ & $\mathbf{C T}$ & $\mathbf{I N}$ & $\mathbf{K B}$ & $\mathbf{P B}$ & $\mathbf{P R}$ & $\mathbf{T F}$ \\
\hline $\mathrm{A}$ & 3 & 64 & 88 & - & 64 & 104 & 64 & 96 \\
$\mathrm{~B}$ & 5 & - & 160 & - & 80 & 136 & 72 & 124 \\
$\mathrm{C}$ & 2 & 64 & - & - & 32 & 48 & 36 & 68 \\
$\mathrm{D}$ & 2 & - & - & - & - & 80 & 48 & 104 \\
$\mathrm{E}$ & 3 & - & - & 300 & - & - & - & - \\
$\mathrm{F}$ & 3 & 48 & 32 & - & 32 & 44 & 24 & 44 \\
$\mathrm{G}$ & 5 & - & - & - & 92 & 152 & 104 & 176 \\
$\mathrm{H}$ & 5 & 88 & 128 & - & 80 & 128 & 48 & 104 \\
$\mathrm{I}$ & 2 & 56 & - & - & 32 & 56 & 32 & 64 \\
$\mathrm{~J}$ & 2 & - & - & 300 & - & - & - & - \\
$\mathrm{K}$ & 5 & 88 & 120 & - & 40 & 88 & 40 & 120 \\
$\mathrm{~L}$ & 3 & 56 & 56 & - & 32 & 64 & 32 & 64 \\
$\mathrm{M}$ & 3 & 72 & - & - & 32 & 80 & 32 & 64 \\
$\mathrm{~N}$ & 2 & 56 & - & - & 40 & 84 & 48 & 68 \\
\hline
\end{tabular}

$\dagger n$ represents number of days an employee can be assigned work. $n=5$ represents a full-time employee whereas other values of $n$ represent part-time employees. The values in this table are obtained from results given in [26].

To compare the quality of solutions obtained by backtracking approaches with previous results, we have used two quality indices namely enumerative skillset index and weighted skillset index that indicate the cumulative expertise of employees in these solutions. The enumerative skillset index, $S_{e}$, is calculated as shown below.

$$
S_{e}=\sum_{e} \sum_{t} E_{e t}
$$

where $E_{e t}$ is the enumerative skill score of an employee ' $e$ ' for task ' $t$ '. These values are obtained by arranging the tasks, that an employee has experience, in increasing order of hours worked and then assigning them integer values starting from 1 . For example, employee $C$ has experience on five tasks: KB (32 hours), PR ( 36 hours), PB (48 hours), AF (64 hours) and TF (68 hours). These tasks are assigned skill scores as 1, 2, 3, 4 and 5.

Although the enumerative skillset index is a good indication of expertise of employees used in a solution, it is not very accurate. Hence, a weighted skillset index, $S_{w}$, is used as shown below.

$$
S_{w}=\sum_{e} \sum_{t} W_{e t}
$$

where $W_{e t}$ is the weighted skill score of an employee ' $e$ ' for task ' $t$ '. These values are obtained by dividing the number of hours an employee ' $\mathrm{e}$ ' has worked on task ' $t$ ' by his/her total work experience in hours. For example, employee $C$ has total experience of 248 hours. Thus, $W_{e t}$ values for tasks KB, PR, 
$\mathrm{PB}, \mathrm{AT}$ and TF having experience of 32, 36, 48, 64 and 68 hours respectively are obtained as $0.129,0.145,0.194,0.258$ and 0.274 .

In this work, backtracking algorithm is applied to this personnel scheduling problem using a PC with $2 \mathrm{GHz}$ processor and $2 \mathrm{~GB}$ RAM. Backtracking is an exhaustive search technique that guarantees optimal solution. However, as the problem under consideration is NP-hard, it is almost impossible to perform an exhaustive search. For example, the personnel-oriented solution tree has a depth of 70 with 9 alternatives at each node. Thus, the search tree will have $70 ! \times 9^{70}$ nodes out of which $9^{70}$ nodes are distinct. Similarly, the task-oriented solution tree has a depth of 45 with 14 alternatives at each node. Thus, this search tree will have 45 ! $\times$ $14^{45}$ nodes with $14^{45}$ distinct nodes. Although the task-oriented representation has smaller search space than the personneloriented representation, it is still too huge for exhaustive search. Hence, all runs of backtracking are executed for fixed time (30 minutes).

A quick way to find good solutions in the early exploration of search process is to use different variable orderings, value orderings and consistency enforcement techniques that will avoid some illegal solutions and quickly generate good solutions. Table 2 shows the results obtained using chronological backtracking with proposed variable and value ordering for both the solution representations. These solutions satisfy all the constraints. Observe that the task-oriented representation has produced better solutions than the personnel-oriented representation. Also, the row-wise (employee or task wise) variable ordering has required less time to produce similar solutions than the column-wise ordering approach.

Table 2 Results obtained using Chronological backtracking with variable and value ordering.

\begin{tabular}{|c|c|c|c|}
\hline $\begin{array}{c}\text { Solution } \\
\text { Represen- } \\
\text { tation }\end{array}$ & $\begin{array}{l}\text { Variable } \\
\text { ordering }\end{array}$ & Value ordering & $\begin{array}{l}\text { Skillset Index } S_{e} \text { and } \\
\text { time in second }\end{array}$ \\
\hline \multirow{6}{*}{$\begin{array}{c}\text { Personnel- } \\
\text { oriented }\end{array}$} & \multirow{3}{*}{$\begin{array}{l}\text { Column } \\
\text { wise } \\
\text { (Day wise) }\end{array}$} & $\mathrm{AF}, \mathrm{CT}, \ldots \mathrm{TF}$ & No solution \\
\hline & & Sorted workload & $174(1)$ \\
\hline & & $\begin{array}{c}\begin{array}{c}\text { Sorted skillset and } \\
\text { workload }\end{array} \\
\end{array}$ & No solution \\
\hline & \multirow{3}{*}{$\begin{array}{l}\text { Row wise } \\
\text { (Emp wise) }\end{array}$} & $\mathrm{AF}, \mathrm{CT}, \ldots \mathrm{TF}$ & $180(1)$ \\
\hline & & Sorted skillset & $174(1), 176(1)$ \\
\hline & & \begin{tabular}{|c|}
$\begin{array}{c}\text { Sorted skillset and } \\
\text { workload }\end{array}$ \\
\end{tabular} & $\begin{array}{c}172(6), 184(6), \\
187(240) \\
\end{array}$ \\
\hline \multirow{2}{*}{$\begin{array}{c}\text { Task- } \\
\text { oriented }\end{array}$} & $\begin{array}{l}\text { Column } \\
\text { wise } \\
\text { (Day wise) }\end{array}$ & $\mathrm{A}, \mathrm{B}, \ldots \mathrm{N}$ & $\begin{array}{c}187(1), 189(2), 190(3), \\
\text { 191(47), 192(130), } \\
\text { 193(510), 194(535) }\end{array}$ \\
\hline & $\begin{array}{l}\text { Row wise } \\
\text { (task wise) }\end{array}$ & $\mathrm{A}, \mathrm{B}, \ldots \mathrm{N}$ & $\begin{array}{c}\text { 187(1), 189(2), 190(4), } \\
\text { 191(46), 192(122), } \\
193(261), 194(272)\end{array}$ \\
\hline
\end{tabular}

The best solution is obtained as shown in Table 3. It has skillset index $S_{e}=194$ and was obtained using task-oriented solution representation with both the column-wise and rowwise variable ordering in 535 and 272 seconds respectively. Note that the best solution obtained using personnel-oriented solution representation has skillset index $S_{e}=187$.
Table 3 Best task schedule obtained using backtracking.

\begin{tabular}{|c|c|c|c|c|c|}
\hline & MON & TUE & WED & THU & FRI \\
\hline A & & KB & & KB & KB \\
\hline B & CT & CT & CT & CT & CT \\
\hline C & PB & & PB & & \\
\hline D & PR & & PR & & \\
\hline E & IN & & IN & & IN \\
\hline F & & AF & & AF & AF \\
\hline G & TF & TF & TF & TF & TF \\
\hline H & PB & PB & PB & PB & PB \\
\hline I & KB & & KB & & \\
\hline J & & IN & & IN & \\
\hline K & TF & TF & TF & TF & TF \\
\hline L & & PR & & PR & PR \\
\hline M & & PB & & PB & PB \\
\hline N & AF & & AF & & \\
\hline
\end{tabular}

Table 4 shows the results obtained using backtracking with consistency enforcement techniques along with the variable and value ordering for personnel-oriented solution representation. The assignment of tasks to part time employee posed some difficulties during backtracking. To avoid them, we have forced that part-time employees should not work on two consecutive days. To further improve the quality of solution and speed up the algorithm, best_task_constraint flag is set which forces the best task allocation to fulltime employees.

Table 4 Results obtained using backtracking with consistency enforcement.

\begin{tabular}{|c|c|c|c|c|}
\hline \multirow{2}{*}{$\begin{array}{c}\text { Solution } \\
\text { Represen } \\
\text { tation }\end{array}$} & \multirow{2}{*}{$\begin{array}{l}\text { Variable } \\
\text { ordering }\end{array}$} & \multirow{2}{*}{$\begin{array}{l}\text { Value } \\
\text { ordering }\end{array}$} & \multicolumn{2}{|c|}{$\begin{array}{c}\text { Skillset Index } S_{e} \text { and time in } \\
\text { second }\end{array}$} \\
\hline & & & $\begin{array}{c}\text { without best_task } \\
\text { _constraint }\end{array}$ & $\begin{array}{l}\text { with best_task } \\
\text { _constraint }\end{array}$ \\
\hline \multirow{6}{*}{$\begin{array}{l}\text { Personnel } \\
\text {-oriented }\end{array}$} & \multirow{3}{*}{$\begin{array}{l}\text { Column } \\
\text { wise } \\
\text { (Day } \\
\text { wise) }\end{array}$} & $\mathrm{AF}, \mathrm{CT}, \ldots \mathrm{TF}$ & $180(1)$ & $185(115)$ \\
\hline & & $\begin{array}{c}\text { Sorted } \\
\text { workload }\end{array}$ & 174(1) & $185(2)$ \\
\hline & & $\begin{array}{l}\text { Sorted skillset } \\
\text { and workload }\end{array}$ & $172(32)$ & No solution \\
\hline & \multirow{3}{*}{$\begin{array}{c}\text { Row wise } \\
\text { (Emp } \\
\text { wise) }\end{array}$} & $\mathrm{AF}, \mathrm{CT}, \ldots \mathrm{TF}$ & 180(1) & No solution \\
\hline & & $\begin{array}{c}\text { Sorted } \\
\text { workload }\end{array}$ & $174(1), 176(2)$ & $\begin{array}{c}\text { 185(1), 191(2), } \\
194(82)\end{array}$ \\
\hline & & $\begin{array}{c}\text { Sorted skillset } \\
\text { and workload }\end{array}$ & $\begin{array}{c}172(5), 184(6), \\
187(380)\end{array}$ & No solution \\
\hline
\end{tabular}

Franses and Post [26] have solved this problem by using bipartite graph model followed by local search for further improvement in quality of solution. They have reported a good solution that has only two violations (assignment of two or more tasks in a week to employees A and L). In [29], we have solved the same problem instance with small variation using genetic algorithm.

Table 5 shows the comparison of solutions obtained using backtracking approach with previous results reported. Note that there is a significant improvement in the skillsets of the solution obtained using backtracking approach over those obtained using hybrid GA [29] which in turn has better solution than that obtained by Franses and Post [26]. 
Table 5 Comparison of results obtained by backtracking algorithm with other approaches.

\begin{tabular}{|l|c|c|}
\hline \multicolumn{1}{|c|}{ Technique } & $\boldsymbol{S}_{\boldsymbol{e}}$ & $\boldsymbol{S}_{\boldsymbol{w}}$ \\
\hline $\begin{array}{l}\text { Two stage algorithm based on } \\
\text { bipartite graphs [26] }\end{array}$ & 98 & 12.536 \\
\hline Hybrid GA [29] & 127 & 13.936 \\
\hline Backtracking Approach & 194 & 14.057 \\
\hline
\end{tabular}

\section{CONCLUSION}

In this paper, we have obtained the solution for the problem of automated scheduling for laboratory personnel $[26,29]$ using backtracking approach. The application of genetic algorithm for this problem [29] had provided a better solution than that reported earlier in [26]. The main objective of this paper was to find whether genetic algorithmic approach had given optimal solution or not. The results obtained in this paper using backtracking algorithm show that there are indeed better solutions for the problem under consideration.

The results also show that the variable and value ordering have strong effect on the exploration of solutions in backtracking approach. Both the solution representations produce good solutions. However, the task-oriented solution representation is observed to give solutions in lesser time. Also, the outcome of the proposed consistency enforcement approach shows significant reduction in time required to obtain solutions.

We are also currently improving out genetic algorithmic approach [29] to obtain better results for this scheduling problem. We also propose to apply the backtracking algorithm to various scheduling problems, particularly for which the genetic algorithm like approaches have been used widely in the recent past.

\section{REFERENCES}

[1] Causmaecker, P.D., Demeester, P., Berghe, G.V., and Verbeke, B. 2004. Analysis of Real-world Personnel Scheduling Problems. 2004. In Proc. of $5^{\text {th }}$ Practice and Theory of Automated Timetabling.

[2] Ernst, A. T., Jiang, H., Krishnamoorthy, M., and Sier, D. 2004. An annotated bibliography of personnel scheduling and rostering. J. Annals of Operations Research. 127, 21144

[3] Ernst, A. T., Jiang, H., Krishnamoorthy, M., and Sier, D. 2004. Staff scheduling and rostering: A review applications, methods and models. J. European Journal of Operational Research. 153, 3-27.

[4] Burke, E. K., Causmaecker, P. D., Berghe, G. V., and Landeghem, H. V. 2004. The state of the art of nurse rostering. J. Journal of Scheduling, 7, 441-499.

[5] Wren, A. 1996. Scheduling, Timetabling and Rostering A Special Relationship? In Proc. of $1^{\text {st }}$ Practice and Theory of Automated Timetabling, 46-75.

[6] Li, J., and Kwan, R.S.K. 2003. A Fuzzy Genetic Algorithm for Driver Scheduling. J. European Journal of Operational Research. 147, 334-344.
[7] Li J., Kwan, R.S.K. 2005. A Self-Adjusting Algorithm for Driver Scheduling. J. Journal of Heuristics. 11, 351-367.

[8] Miyashita, T. 2003. An Application of Immune Algorithms for Job-Shop Scheduling Problems. In Proc. of the $5^{\text {th }}$ IEEE International Symposium on Assembly and Task Planning.

[9] Jensen, M.T. 2003. Generating Robust and Flexible Job Shop Schedules Using Genetic Algorithms. J. IEEE Transactions on Evolutionary Computation. 7, 275-288.

[10] Ombuki B.M., and Ventresca, M. 2004. Local Search Genetic Algorithms for the Job Shop Scheduling Problem. J. Applied Intelligence. 21, 99-109.

[11] Wilke, P., Grobner, M., and Oster, N. 2002. A Hybrid Genetic Algorithm for School Timetabling.

[12] Karova, M. 2004. Solving Timetabling Problems Using Genetic Algorithms. Proc. of $27^{\text {th }}$ Int'l Spring Seminar on Electronic Technology.

[13] Qu R., and Burke, E. K. 2005. Hybrid Variable Neighborhood HyperHeuristics for Exam Timetabling Problems. In Proc. of $6^{\text {th }}$ Metaheuristics International Conference.

[14] Kumar, V. 1992. Algorithms for Constraint Satisfaction Problems: A Survey. AI Magazine. 13(1), 32-44.

[15] Bartak, R. Constraint propagation and backtracking based search.

[16] Horowitz, E., Sahni, S., and Rajasekaran, S. 2008. Fundamentals of Computer Algorithms. Universities Press.

[17] Dechter, R., and Frost, D. 2002. Backjump-based backtracking for constraint satisfaction problems. J. Artificial Intelligence. 136, 147-188.

[18] Musliu, N. 2001. Intelligent search methods for workforce scheduling: New ideas and practical applications. $\mathrm{PhD}$ thesis. Fakultat Fur, Tecnische Universitat Wien, Vienna, Austria.

[19] Jegou, P., and Terrioux, C. 2003. Hybrid backtracking bounded by tree decomposition of constraint networks. J. Artificial Intelligence. 146, 43-75.

[20] Sadeh, N., Sycara, K., and Xiong, Y. 1995. Backtracking techniques for the job shop scheduling constraint satisfaction problem.

[21] Lau, H. C. 1996. Combinatorial approaches for hard scheduling problems in manpower scheduling. J. Operations research. 39(1)

[22] Brailsford, S. C., Potts, C. N., and Smith, B. M. 1999. Constraint satisfaction problems: Algorithms and applications. J. European Journal of Operational Research. 119, 557-581.

[23] Kondrak, G. 1994. A Theoretical Evaluation of selected backtracking algorithms. Thesis Master of Science, Edmonton, Alberta. 
[24] Prosser, P. 1993. Domain filtering can degrade intelligent backtrack search. In Proc. of the International Joint Conference on Artificial Intelligence, 262-267.

[25] Prosser, P. 1993. Forward checking with backtracking. Technical report AISL 48-93, University of Strathclyde.

[26] Franses, P., and Post, G. 2002. Personnel Scheduling in Laboratories. In Proc. of $4^{\text {th }}$ Practice and Theory of Automated Timetabling, 113-119.

[27] Sadeh, N. M, and Fox, M. S. 1995. Variable and value ordering heuristics for the job shop scheduling constraint satisfaction problem. Technical report CMU-RI-TR-9539.

[28] Mackworth, A.K., and Freuder, E.C. 1985. The Complexity of some Polynomial Network Consistency Algorithms for Constraint Satisfaction Problems. J. Artificial Intelligence. 25, 65-74.
[29] Adamuthe, A. C., and Bichkar, R. S. 2011. Genetic algorithmic approach for personnel timetabling. In Proc. of International Conference on Technology Systems and Management 2011, 69-76, Springer- Verlag.

[30] Burke, E.K., Cowling, P., Causmaecker, P.D., and Berghe, G.V. 2001. A Memetic Approach to the Nurse Rostering Problem. J. Applied Intelligence. 15, 199-214.

[31] Ozcan, E. 2005. Memetic Algorithms for Nurse Rostering. In Proc. of 20th International Symposium on Computer and Information Sciences, 482-492.

[32] Maenhout, B., and Vanhoucke, M. 2006. A Comparison and Hybridization of Crossover Operators for the Nurse Scheduling Problem. Working Papers of Faculty of Economics and Business Administration, Ghent University. 